\title{
GOBIERNO CORPORATIVO Y DESEMPEÑO DE LA EMPRESA CHILENA. EVIDENCIA EMPÍRICA POR MEDIO DE DATOS DE PANEL
}

\section{GONZALO ALTAMIRANO CARRASCO}

- Facultad de Ciencias Económicas y Administrativas Universidad Católica de la Santísima Concepción, Chile.

\section{KATHERINE CARTES MATUS}

- Facultad de Ciencias Económicas y Administrativas Universidad Católica de la Santísima Concepción, Chile.

\section{PABLO SAN MARTÍN MOSQUEIRA ${ }^{a}$}

- Facultad de Ciencias Económicas y Administrativas Universidad Católica de la Santísima Concepción, Chile.

RESUMEN

Este documento investiga la relación entre la estructura de propiedad y el directorio con el valor de la empresa. La muestra consiste en un panel de 85 empresas chilenas no financieras para el periodo 2006-2013. Los resultados son consistentes con investigaciones previas para el caso chileno. La estructura de propiedad y la pertenencia a un grupo económico tienen influencia en el valor de la empresa. Los resultados del análisis econométrico evidencian una relación negativa entre estructura de propiedad y valor de empresa, sin embargo existe una relación positiva cuando la firma es parte de un grupo económico y aumenta en el tamaño del directorio, ambos resultados son estadísticamente significativos.

Palabras Claves: Gobierno corporativo, Estructura de propiedad, Grupos económicos, Datos de panel.

ABSTRACT

This paper investigates the relation between ownership structure and boards with the value of the company. The sample consists of 85 Chilean non financial firms for the period 2006-2013. The results are consistent with previous research in the chilean case. The ownership structure and membership of a business group influence the value of the company. The results of the econometric analysis show a negative relation between ownership structure and firm value, however there is a positive relation when the firm is part of a business group and increases in the size of the board, both results are statistically significant.

Key words: Corporate governance, Ownership structure, Business group, Panel data.

El buen gobierno corporativo se ha vuelto cada vez más importante para el desarrollo del mercado financiero, toda vez que, tanto a nivel local como internacional, se suceden numerosos fraudes financieros.

\section{INTRODUCCIÓN}

Un buen gobierno corporativo da respuesta a los principios sobre los cuales funcionan los primordiales actores en una empresa, como son: los accionistas, el directorio y la alta administración. El valor de una empresa se puede ver afectado por su estructura de propiedad y por su directorio.

A su vez, existen mecanismos de controles legales y de autorregulación, a fin de mejorar las prácticas de los gobiernos corporativos. Todo esto se gesta en pos de dar confianza en el mercado y porque se reconoce una relación entre el buen manejo de los gobiernos corporativos y el valor de esa empresa en el mercado. 
El objetivo de la presente investigación es determinar la influencia de mecanismos internos de gobierno corporativo (estructura de propiedad y composición del directorio) en el valor de la empresa, no financiera, cotizada chilena. Esta temática ha recibido relativamente poca atención en el mercado chileno, por lo que su análisis se vuelve muy relevante. El análisis se desarrolla para un panel compuesto por una muestra de 85 empresas chilenas, para el período comprendido entre los años 2006 y 2013.

El estudio se organiza de la siguiente forma: La sección 2 contiene una revisión de la literatura y plantea las hipótesis de estudio. La sección 3 presenta la muestra, variables, modelos utilizados y metodología de análisis. Los resultados se presentan en la sección 4 y, finalmente, la sección 5 resume las principales conclusiones de este estudio.

\section{REVISIÓN DE LA LITERATURA}

Luego de la caída de diversas corporaciones en el mundo como Enron, WorldCom y Parmalat' ${ }^{1}$, hace un par de décadas, nos encontramos nuevamente con corporaciones que han tenido un mal desempeño en materias de gobiernos corporativos, en desmedro de la estabilidad y buen funcionamiento del sistema financiero, como es el ejemplo de Lehman Brothers ${ }^{2}$. Al revisar los antecedentes de todas estas compañías, podemos ver que siguen un patrón similar, solo cambian los actores. Chile, economía emergente latinoamericana, no se encuentra ajena a esta temática. Solo por nombrar algunas compañías chilenas que pasaron por problemas similares, encontramos a Enersis con el llamado caso "Chispas" y recientemente el caso La Polar. El análisis de las consecuencias de estos sucesos pone en cuestión la efectividad de la administración de las empresas, dejando entrever la necesidad de más y mejores normativas y que estas sean más efectivas.

El trabajo de Berle y Means (1932), que pone de manifiesto los problemas ocasiones por la dispersión en la propiedad de una compañía y los problemas de control que esta genera, que trae como consecuencia el problema de agencia. Hoy en día esta separación es común en las empresas, presentando quizás una ventaja; la propiedad puede cambiar de manos sin interferir con la operación de la empresa (Casassus et al, 2010).

No fue sino hasta que el trabajo de Jensen y Meckling ve la luz en el año 1976, que se formaliza la idea de que los directivos (agente) de una empresa persiguen objetivos distintos a los accionistas (principal). Dado que los accionistas poseen un derecho residual sobre las utilidades, entonces ante posibles conflictos entre el agente y principal, como consecuencia de las asimetrías de información entre estos, los directores actúan en su propio beneficio en desmedro del beneficio de los propietarios, conflictos que tienen su efecto sobre el valor de la empresa, disminuyéndolo, debido a los costos de agencia ${ }^{3}$.

Por consiguiente, cuando la empresa le pertenece a un único dueño y sobre este recae la administración de la compañía, no existirán costos de agencia. Costos que aumentan cada vez que la propiedad de la empresa se encuentra más atomizada.

Para Roqueiro (2010) el problema de agencia no solo afecta a la empresa, sino que también afecta negativamente a la eficiencia de una economía. A modo de ejemplo, se puede mencionar el encarecimiento de recursos financieros y dado que el problema aumenta con la dispersión de capital, será necesario mantener un porcentaje del capital en manos de los directores, alineando así sus intereses con los de los accionistas.

Shleifer y Vishny (1997), definen gobierno corporativo "como la forma en que los proveedores de financiamiento para las empresas se aseguran de obtener un retorno de su inversión". Por otra parte, John y Senbet (1998) definen de forma más completa gobiernos corporativos, establecen que "son los mecanismos mediante los cuales los stakeholders ejercen control sobre los administradores de la empresa de tal manera que se les protejan sus intereses". Para Lefort (2008) gobierno corporativo puede ser definido como el "conjunto de instancias y prácticas institucionales que influyen en el proceso de toma de decisiones de una empresa, contribuyendo a la creación sustentable de valor en un marco de transparencia y responsabilidad empresarial".

En concordancia con lo que plantea Jensen y Meckling (1976), Hart (1995) explica que hay dos condiciones que están presentes cuando hay problemas en los gobiernos corporativos. "En primer lugar, que exista problema de agencia o conflicto de intereses y, en segundo lugar, los costos de transacción sean tales que este problema de agencia no pueda ser tratado a través de un contrato".

No obstante, una visión mucho más formalizada del concepto de Gobierno Corporativo es la que entrega la OCDE (2004), que lo define como el "conjunto de prácticas y mecanismos que proveen incentivos apropiados al directorio y al grupo ejecutivo para proteger los intereses de la compañía y de sus accionistas y que facilitan el control efectivo de la gestión de la empresa, de tal modo de incentivar la creación de valor y el uso eficiente de recursos".

Independiente del significado que le asignan una vasta gama de autores a este término, es preciso mencionar que todos concuerdan en que se busca maximizar el valor de la empresa, alineando los intereses de los accionistas y directivos para minimizar los costos asociados. Además podemos notar que no solo los accionistas se benefician de tener altos estándares y un buen gobierno corporativo, sino que también el mercado e intermediarios de valores entre otros.

La Superintendencia de Valores y Seguros (SVS), entidad supervisora del mercado de valores en Chile, formula una serie de estándares mínimos en materia de buen gobierno corporativo, según las que se espera: a) se establezcan y revisen periódicamente las políticas y estrategias de la empresa; b) supervisar a la alta gerencia en el cumplimiento de sus funciones; c) establecer políticas adecuadas de remuneración y compensaciones; d) establecer códigos de ética; e) Implementar un adecuado sistema de control del flujo de información. 


\subsection{Mecanismos de Gobierno Corporativo}

En mercados más desarrollados y eficientes, como los de Norteamérica y Europa, con instituciones que protegen a los inversionistas, encontraremos empresas cada vez más dispersas en cuanto a propiedad y control. Esto genera, a la vez, que los accionistas deban delegar cada vez más responsabilidades en diversos ejecutivos, los cuales deben maximizar la riqueza de la empresa y, por consecuencia, la de sus propietarios. Como no es posible monitorear todo el trabajo realizado por los ejecutivos, se hace necesario e imprescindible establecer mecanismos de control para mitigar los problemas de agencia y riesgo moral, derivado de contratos incompletos y de intereses contrapuestos (Hölmstrom, 1979). Estos mecanismos de control pueden ser internos y externos.

\subsubsection{Mecanismos de control interno}

Corresponden a distintas herramientas con las que se dispone al interior de la empresa y que ayudan al buen funcionamiento de su gobierno corporativo.

Directorio: La función principal de todo directorio es ser el intermediario entre los accionistas y la administración. Es labor del directorio guiar, supervisar y controlar la administración, representando y defendiendo los intereses de todos los accionistas y, por otro lado, comunicando a los propietarios las propuestas de la administración.

En el directorio de la empresa se encuentran tanto directores dependientes como independientes. Una de las características del director independiente es que no tiene vínculo con los propietarios ni con los directores ejecutivos internos de una determinada empresa, otra característica es que se pueden encontrar en más de una empresa como director. Para Fama y Jensen (1983) estos no tienen incentivo de expropiar rentas, sino que su principal motivación es obtener una mayor reputación por su trabajo, por ello su rol es fundamental para supervisar y ejercer un mayor control sobre los directores ejecutivos. Además, y prueba de lo anterior, es que mientras existan más directores independientes en una empresa, la probabilidad de manipulación de cifras contables sería cada vez menor (Peasnell et al. 2001; Klein 2002). Además, la evidencia da cuenta de que una mayor proporción de directores independientes se relaciona positivamente con una mayor valoración de mercado para la empresa (Lefort y Urzúa, 2008).

Estructura de propiedad: En las empresas la propiedad y el control parecieran ser sinónimos, pero en realidad dependen de: (1) el grado de concentración accionarial, determinando que mientras más concentrada se encuentre la propiedad mayor control ejerce el accionista mayoritario / controlador, y (2) si los individuos que toman el control de la empresa son individuos particulares o familias. (Fama y Jensen, 1983).

En estructuras de propiedad, por tanto, el accionista controlador tiene gran relevancia en temas de control. Por otra parte, lo anterior da pie a que existan de igual manera otros accionistas, minoritarios, los cuales son también propietarios aunque los niveles de propiedad que manejan no les permita ejercer el control.

Endeudamiento: El uso de la deuda como mecanismo de control fue explicado por Jensen y Meckling (1976). Financiar los recursos de la empresa con deuda impone mayor disciplina en la gerencia. Sin embargo, la gestión de los administradores ya no solo es evaluada por los accionistas, ahora está bajo la supervisión de los bonistas o acreedores financieros, mejorando con ello la supervisión de la empresa y, es de esperar también, un mejor desempeño. En definitiva alinea los intereses de la gerencia con los propietarios.

Llegado este punto se hace relevante mencionar los costos de agencia, entre accionistas y acreedores financieros: (1) Pérdida de riqueza, dado el uso de la deuda en las decisiones de inversión; (2) Monitoreo constante de los acreedores financieros y (3) Costos de quiebra (Jensen y Meckling 1976).

\subsubsection{Mecanismos de control externo}

Dentro de los mecanismos de control externo encontramos una serie de factores que influyen en el funcionamiento de los gobiernos corporativos. Dentro de los más relevantes se encuentran la competencia del mercado, el sistema legal que enmarca el funcionamiento de estos y el rol de auditoras u otras entidades, tales como instancias de supervisión de mercado como la SVS en Chile, por ejemplo, que con sus normativas y reglamentos sugieren el actuar de los gobiernos corporativos.

Competencia de Mercado: La industria en la cual operan las empresas es un mecanismo natural de control externo. Si las empresas están insertas en industrias competitivas y con estructuras eficientes, tanto operacional como organizacionalmente, la gerencia tendrá mayor presión por obtener mejores resultados, para no dañar la posición de la empresa (Hart, 1983). La rivalidad en las industrias incentiva cada vez a los administradores a mejorar la capacidad de la empresa (Jensen, 1986).

Shleifer y Vishny (1997), reconocen que la competencia de mercado es una fuerza poderosa que genera eficiencia en el mercado, pero que por sí sola no soluciona el problema de los gobiernos corporativos en las empresas.

Sistema Legal: Mucho del marco regulatorio internacional responde a hechos puntuales que remecen la opinión pública y pasan a transformarse en una inquietud en el país de ocurrencia. En Estados Unidos, por ejemplo, se promulgó la Ley SarbanesOxley en 2002, en respuesta al caso Enron, el cual fue conocido por los rumores de presiones políticas y sobornos. Esto dio como resultado una empresa en quiebra, ejecutivos juzgados y una ley que partió con fuerza desde ese entonces y se ha perfeccionado a lo largo del tiempo. 
La Porta, López-de-Silanes, Shleifer y Vishny (1999) manifiestan la importancia del marco legal y de los estándares de gobiernos corporativos, ya que una mala protección a los accionistas es penalizada con bajas valoraciones de la empresa. Por otro lado Chong y López-de-Silanes (2007) en un estudio para América Latina, determinan que los inversionistas tienen un baja protección a sus derechos principalmente por que las leyes establecidas en algunos países (Colombia y Venezuela), derivadas del sistema jurídico del país en cuestión, generan ciertos vacíos legales. Además estas debilidades, en el mercado de capitales, resaltan aún más por las bajas penas o bien por la débil o nula aplicación de la ley. Los autores determinan que Chile y Brasil son los países con mayores reformas legales en materias de gobiernos corporativos en los últimos 30 años en el contexto latinoamericano.

Auditoras: El rol de las auditoras externas es de vital importancia, toda vez que su independencia de juicio y la confidencialidad de la información que manejan, aporta al control y buen desempeño de los gobiernos corporativos.

En Chile, la Ley 20.382 de gobiernos corporativos, incorpora un título aparte para describir las principales funciones de las auditoras externas, velando por la independencia de juicio y la confidencialidad de la información. La ley señala que sus funciones principales se resumen en fiscalizar, controlar y supervisar a las empresas sujeto de ser auditadas.

\subsection{Gobierno Corporativo en Chile}

En Chile existen casos emblemáticos que atañen a los gobiernos corporativos de empresas de gran importancia. Estos hechos han dado pie que tanto en el ámbito legal, como en las prácticas de las empresas, mejore el funcionamiento de los gobiernos corporativos.

En un estudio de la Asociación de AFP de Chile (mayo 2008), se muestra un resumen de los casos más relevantes en materia de gobiernos corporativos. La tabla 1 presenta este resumen.

\section{Tabla 1: Casos emblemáticos chilenos en materia de gobiernos corporativos}

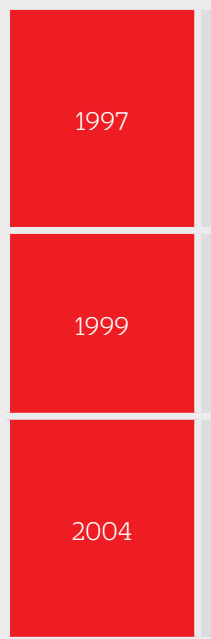

\section{Caso Chispas}

Ejecutivos de ENERSIS y ENDESA España fueron cuestionados por la adquisición y control de ENDESA Chile. El caso Chispas, es uno de los casos más emblemáticos dentro de las operaciones societarias en Chile en las que el tema del gobierno corporativo es central, es además, uno de los primeros y, quizás el que instaló el tema de la importancia del buen gobierno corporativo en Chile

\section{Venta de Telefónica Net a Terra}

El precio ofrecido por Terra para adquirir Telefónica Net estaba por debajo del valor aceptable de mercado. Los directores elegidos por las AFP (Administradoras de Fondos de Pensiones) se opusieron a la transacción y votaron en contra de la operación en Junta de Accionistas. Se iniciaron acciones legales contra Telefónica CTC.

\section{Venta del Negocio Celular a Telefónica Móviles}

Las AFP solicitaron la realización de una Junta Extraordinaria de Accionistas para modificar los términos de una transacción entre Telefónica CTC y su empresa relacionada Telefónica Móviles S.A. La presión de las AFP llevó a un incremento en el precio de venta de 50 millones de dólares y el pago de un dividendo extraordinario a los accionistas de US $\$ 800$ millones

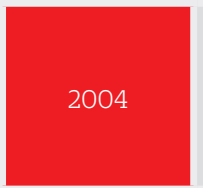

\section{Fusión MASISA - Terranova}

Las AFP negociaron la relación de canje y condicionaron la aprobación de la fusión entre Terranova y MASISA al pago de un dividendo extraordinario de 54 millones de dólares y la baja incidencia del derecho a retiro causado en la operación.

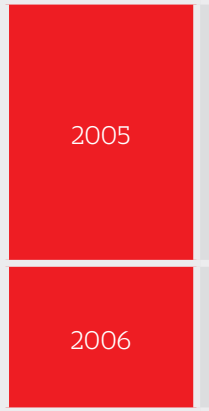

\section{Cambio de Estatutos de Soquimich}

Este ejemplo muestra el rol de las AFP como accionista minoritario significativo en la pugna por el control de SQM. En este caso, las AFP apoyaron a Potash Corporation para modificar los estatutos de SQM y lograr la unificación de los derechos de las dos series accionarias y la imposición del límite máximo de votación de 37,5\% del derecho de voto de cada serie a las votaciones realizadas por accionistas relacionados actuando en pos de un mismo interés.

\section{Pacto Pampa Calichera - Kowa}

Las AFP objetaron a la SVS algunas de las cláusulas de un pacto de accionistas diseñado para lograr el control de SQM. Dichas cláusulas fueron eliminadas.

Fuente: Obtenido de Asociación de AFP (2008).

A estos casos debe sumarse, por ejemplo, el de La Polar (2011) cuyas repercusiones se escuchan hasta el día de hoy, en donde todos los actores (empresarios, accionistas, directores, fiscalizadores, etc.) fueron cuestionados por falta de rigurosidad al no 
detectar a tiempo las repactaciones unilaterales. Como consecuencia de ello se manipularon los estados financieros, y esto permitió a la empresa tener una buena valorización de mercado.

Es así como existe un interés en proteger al accionista minoritario, y la manera de conseguirlo es lograr que los gobiernos corporativos funcionen de manera correcta. En Chile la normativa se ha perfeccionado en virtud de proteger a la parte más débil, promover la transparencia y la entrega de información para un mercado con menos imperfecciones. En la actualidad se cuenta con órganos reguladores tales como las Superintendencias de Valores y Seguros (SVS), la Superintendencia de Administradoras de Fondos de Pensiones (SAFP) y la Superintendencia de Bancos e Instituciones Financieras (SBIF), los que aportan con normativa y reglamentos que apoyan a la misma causa.

Lo anterior ha redundado en la incorporación de reformas al mercado de capitales, al sistema bancario, al de las AFP y a la normativa legal chilena. La participación de empresas chilenas en mercados internacionales, como el norteamericano, obligan a estas compañías a someterse a la legislación de aquellos países. También influye positivamente el hecho de que Chile sea parte de la OCDE y deba cumplir con estándares de gobiernos corporativos, así como otras imposiciones que permiten una mejor imagen y riesgo país.

En cuanto al marco regulatorio chileno se ha perfeccionado con el tiempo. Tal como ha ocurrido en otros contextos, mucha de las reformas han surgido a partir de escándalos financieros que han afectado a grandes compañías, y como consecuencia de ello se ha visto afectado el mercado en general. Tal como lo indica Chong y López-de-Silanes (2007), la historia política y social en Latinoamérica ha retrasado la preocupación en los mercados de capitales, y por ende, la inquietud de mejorar su funcionamiento por lo que no van al mismo ritmo que los mercados Asiáticos o Europeos.

Chile, no obstante, comenzó tempranamente a legislar en este ámbito. El año 1997, la aparición del caso Chispas dio pie a que por vía legal; se mejorara la transparencia, se diera más protección a los accionistas minoritarios, se perfeccionaran los mecanismos de control y se establecieran sanciones más estrictas. Todo esto quedó refundido en la Ley 19.705, del año 2000. A partir de eso han surgido diferentes cuerpos legales que perfeccionan al anterior, en pos de mejorar el sistema. La tabla 2 presenta la regulación vigente.

\section{Tabla 2: Leyes y normativa chilena sobre gobierno corporativo y funcionamiento del mercado}

- Ley 19.705 - 2000: Regula las Ofertas públicas de adquisición de acciones (OPAS) y establece régimen de gobierno corporativo. Conocida como "Ley de OPAS y Gobiernos Corporativos"

- Ley 19.768 - 2001: Introduce adecuaciones de índole tributario al Mercado de Capitales y flexibiliza el mecanismo de ahorro voluntario. Llamada "Ley del Mercado de Capitales I"

- Ley 20.190 - 2007: Introduce adecuaciones tributarias e institucionales para el fomento de la industria de capital de riesgo y continúa el proceso de modernización del mercado de capitales. Es la denominada "Ley de Mercado de Capitales II".

- Ley 20.382 - 2009: Introduce perfeccionamiento a la normativa que regula los gobiernos corporativos de las empresas. Llamada "ley de Gobiernos Corporativos"

- Ley 20.448 - 2010: Introduce una serie de reformas en materia de liquidez, innovación financiera e integración del Mercado de Capitales. Conocida como "Ley de Capitales III".

- NCG 341, de la SVS. Esta Norma General de la SVS se refiere a las prácticas de gobierno corporativos adoptadas por las sociedades anónimas abiertas.

En la Ley 18.046 del año 1981 se indica que "Los directores deberán emplear en el ejercicio de sus funciones el cuidado y diligencia que los hombres emplean ordinariamente en sus propios negocios y responderán solidariamente de los perjuicios causados a la sociedad y a los accionistas por sus actuaciones dolosas o culpables". En este extracto se distingue, que desde un principio se tuvo en cuenta la importancia del buen funcionamiento de los gobiernos corporativos, sin embargo ha sido necesario mejorar las atribuciones y prohibiciones de éstos.

Como ya se indicó, hace unas décadas atrás la discusión en Chile sobre el uso y manejo de información privilegiada no era un tema que preocupara, toda vez que las condiciones políticas y sociales parecían abarcar todas las miradas. En la actualidad, dadas las recientes reformas, ello ha cambiado bastante.

Según un informe elaborado por el Banco Mundial en el año 20114, "Chile, con su modificación de la ley de mercados de valores, fortaleció la protección de inversores, al imponer una mayor divulgación de información corporativa y regular la autorización de transacciones entre partes vinculadas." 
En relación a esto último, el año 2012 sólo 11 empresas chilenas lograron cumplir con los estándares de transparencia superando el mínimo internacional (Índice de Transparencia Corporativa). Según lo publicado por KMPG las empresas que lograron el estándar fueron: Masisa, Banco Santander, Banco BCI, Banco de Chile, Antofagasta Minerals, Endesa, LAN, E-CL, Aquachile y Aguas Andinas. Esto demuestra que grandes empresas buscan cumplir con los estándares internacionales con el fin de mejorar su imagen, entendiendo que la mayoría participa en dichos mercados.

Todo lo anterior, vuelve a poner de manifiesto la importancia del funcionamiento de los directorios en la empresa y a poner atención en la manera en que se protege a los accionistas minoritarios.

\subsection{Hipótesis}

A continuación se exponen las hipótesis para esta investigación, las que se centran en la estructura de propiedad y directorio; como variables que inciden en el valor de empresa.

Una serie de estudios relacionan la estructura de propiedad con el desempeño de la empresa. El trabajo pionero de Berle y Means (1932) propone esta avenida de estudio y da sustento a una extensa cantidad de literatura en la que se muestra la influencia que tiene la forma en que se estructura la propiedad societaria sobre el valor de la empresa.

A pesar de la vasta literatura no se ha logrado un consenso sobre cómo afecta la estructura de propiedad al desempeño de la empresa, ya que se pueden encontrar relaciones positivas y negativas.

Cuando la estructura de propiedad se encuentra muy concentrada, en un accionista dominante, este se atribuye un gran control sobre la organización. Ello lleva a que éste tenga incentivos para expropiar riqueza de los accionistas minoritarios, generando un impacto negativo en la empresa., efecto conocido como atrincheramiento (Demsetz, 1983 y Fama y Jensen, 1983). Así, Boubaker (2007) para una muestra de 510 empresas francesas, y utilizando como medida de desempeño la $Q$ Tobin, verificó que el control de la propiedad afecta el valor de la empresa en forma negativa. Mismo estudio que también realizó Pedersen y Thomsen (2001), quienes postulan que los accionistas controladores tienen incentivos a expropiar riquezas en desmedro de los accionistas minoritarios.

Caso contrario se da cuando la concentración de propiedad provoca un mayor y mejor control y monitoreo en forma positiva. Esto reduce los costos de agencia y genera un mayor valor de la empresa (Shleifer y Vishny, 1986). Existe evidencia, por tanto que, da cuenta de una relación positiva entre valor de empresa y concentración de la propiedad (Fuerst y Kang 2000; Morck et al 1988; McConnell y Servaes 1990).

Para el caso de Chile hay estudios con evidencia sobre estructura de propiedad y su influencia en el valor de la empresa. Raineri y Valenzuela (2001) evidencian que en el mercado chileno el porcentaje de propiedad en manos del primer accionista está entre un 40\% y 50\%, y que el control de la empresa podría estar siendo dominado por una sola persona o familia. Lefort y Walker (2005), tras verificar una alta concentración de propiedad en los conglomerados chilenos, encuentran que el desempeño de la empresa y la estructura de propiedad se encuentran relacionados negativamente. Espinosa (2009), para 204 empresas chilenas entre 1998 y 2007, encuentra evidencia negativa y significativa entre concentración de la propiedad y desempeño.

Así mismo, Espinosa y Maquieria (2010), para una muestra de 70 empresas chilenas, entre los años 2000 y 2007 , verifican una relación negativa y significativa para el principal y los tres principales accionistas con el desempeño de la empresa. Sin embargo, a través de sus resultados es posible verificar la existencia de una relación no lineal entre estructura de propiedad y desempeño, específicamente con una forma de “U”, reportada también previamente por Maquieira y Espinosa (2006).

Respecto a la evidencia empírica para Chile, podemos establecer nuestra primera hipótesis de investigación.

\section{H1: “Una alta concentración de la propiedad tiene una influencia negativa en el valor de la empresa”.}

Como se expuso en el párrafo anterior, Chile presenta una alta concentración de propiedad y una estructura corporativa dominada por conglomerados. En países latinoamericanos el desarrollo de conglomerados ha servido para eludir las ineficiencias locales, permitiendo a través de ellos usar sus propios recursos de inversión y servicios que no están disponibles en el mercado local (Raineri y Valenzuela, 2001).

Lefort y Walker (2000), reportan que el 70\% de las empresas pertenecen a algún conglomerado y estas representan aproximadamente un $90 \%$ de los activos de las empresas no financieras chilenas. Cabe destacar que las empresas que pertenecen algún conglomerado generaran sinergias tanto operacionales como financieras, transfiriendo y compartiendo recursos dentro del grupo.

Raineri y Valenzuela (2001) evidencian una débil correlación entre el desempeño de las empresas y la pertenencia a algún conglomerado, que con el correr de los años esta es cada vez menor. Lefort y Urzúa (2008), entre los años 2000 y 2003 , para empresas no financieras chilenas, encuentran que un 70\% de las empresas pertenecen a algún conglomerado, evidenciando que si la empresa pertenece a alguno y, además posee directores profesionales, mejoran el desempeño de la empresa. Por consiguiente, formulamos nuestra segunda hipótesis de investigación: 


\section{H2: "La pertenencia de la empresa en un grupo económico, tiene una influencia positiva en el valor de la empresa”.}

Al revisar la estructura propiedad, no podemos quedar indiferentes ni dejar de mencionar al rol que ejercen los inversionistas institucionales en Chile, en especial las AFP. Como se mencionó en la sección anterior, otro enfoque del problema de agencia se puede presentar como el conflicto de interés entre accionistas mayoritarios y minoritarios.

En la década de los años 80, cuando se le autorizó invertir a los fondos de pensiones en sociedades anónimas, se inició una serie de procesos de cambios regulatorios a fin de mejorar este sistema. Actualmente las AFP administran recursos por un monto de 135.000 millones de dólares ${ }^{6}$, distribuidos, aproximadamente, un 40\% en renta variable y un 60\% en renta fija.

La labor de las AFP en Chile es importante, ya que la alta concentración de propiedad de las empresas y el control de estas por parte de los grupos económicos bajo estructuras piramidales de las compañías, inciden en la creación de problemas de gobiernos corporativos. Es por ello que las AFP deben ir en defensa del ahorro previsional de sus afiliados, protegiendo los intereses de los accionistas minoritarios de las sociedades en las que participan y de los tenedores de bonos.

Cabe mencionar que las AFPs son el principal inversionista del mercado financiero chileno. Al ser este un mercado poco profundo, cuando las AFPs invierten en una empresa dan una señal para quienes participan en él. El controlador es el principal beneficiado de que el precio de sus acciones aumente y que estos papeles sean más líquidos. La señal que entregan, al ser las AFPs inversionistas especializados y con mayor información que los demás agentes del mercado, es invertir en empresas con un alto valor y proyección (Tong y Ning 2004).

Walker y Lefort (2000) para un estudio para Chile evidencian que, como efecto de las reformas introducidas en el país, las empresas han disminuido su costo de capital. En concordancia con lo expuesto anteriormente Lefort y Walker (2007) muestran que las empresas en las que las AFP invierten, valen un 14\% más que aquellas en las que no invierten. Ello, entonces, permite establecer nuestra tercera hipótesis de investigación:

\section{H3: "La participación de las AFP en la propiedad de la empresa tiene una influencia positiva sobre su valor".}

Tal como se mencionó anteriormente, el marco que regula las AFP en Chile, se ha ido perfeccionando en el tiempo, con una tendencia a proteger al accionista minoritario. En este contexto, el Decreto Ley 3.500, considera que debe existir una preocupación de las AFP respecto al gobierno corporativo, toda vez que en ellas se invierten los recursos de los afiliados al sistema previsional chileno.

El directorio de las AFPs es responsable de la elección de candidatos a directores en aquellas sociedades en las que están invirtiendo sus fondos. Estos deben votar a viva voz en las elecciones de directores y pronunciarse en materias de acuerdos.

Por esta razón, las AFP deben cautelar su independencia frente a los candidatos a director de una sociedad. La intensión de la legislación es que la AFP no participe activamente en la administración de la empresa. Para ello la ley ha determinado un 7\% como el máximo de acciones suscritas con recursos de las administradoras.

Tal como se comentó, la evidencia internacional indica que las empresas que tienen entre sus accionistas la presencia de las fondos de pensiones, tienden a ser más valoradas por el mercado, ya que estas ejercen un rol de monitoreo.

Ello da pie a nuestra cuarta hipótesis de investigación, la cual es:

\section{H4: "La participación de las AFP en el directorio de la empresa tiene una influencia positiva sobre el valor de la empresa".}

En empresas altamente concentradas, los directores son frecuentemente elegidos por los accionistas controladores. Esto provoca una divergencia de intereses por que los directores tendrán influencia en las decisiones que favorecen a los accionistas mayoritarios. Para subsanarlo existe una tendencia a utilizar buenas prácticas de gobierno corporativo, las cuales buscan generar instancias e incluir un mayor número de directores independientes en la toma de decisiones para que estos busquen la maximización de riqueza de todos los accionistas y no solo quede en manos de unos cuantos.

Existe literatura sobre la influencia de los directorios en el desempeño de la firma. Dechow et al (1996), Peasnell et al. (2001), Klein (2002) y Xie et al (2003) concluyen que un mayor número de directores independientes, reuniéndose frecuentemente, contribuyen a una mayor integridad en el directorio, reduciendo con ello la asimetría de información, mejorando y otorgando mayor resguardo a los intereses de los accionistas minoritarios. Por otro lado, Lei y Song (2012), encuentran evidencia significativa para Hong Kong, la que se refiere a que una estructura directiva es fundamental para el valor de la empresa, y se demuestra que a mayor porcentaje de directores independientes, mayor es el valor para dicha empresa. Esto nos permite enunciar la quinta hipótesis de investigación:

\section{H5: "Un mayor porcentaje de directores independientes dentro del directorio tiene un efecto positivo sobre el valor de la empresa".}

El tamaño del directorio, medido como el número de directores en la empresa, influye positivamente en la supervisión de la administración. Castro et al (2009) analizan el directorio de las empresas de Brasil, Chile y México, encontrando que Chile posee el directorio con menos número de integrantes con 8.08 directores por junta, para Brasil y México son 8.93 y 11.69 respectivamente. 
Un directorio elevado, genera diversas opiniones y un mayor control en las acciones de la administración de la empresa. Observando los contras, podemos indicar que un número elevado de directores podría volverse ineficiente en la toma de decisiones por la diversidad de posturas y por la falta de coordinación. Es así como el valor de la empresa podría verse beneficiado o en caso contrario, perjudicado. No hay un claro consenso en relación a un número efectivo de directores.

Yermack (1996) para un periodo comprendido entre 1984 y 1991, encuentra una relación negativa entre valor de empresa medido a través de la Q Tobin y el tamaño del directorio, siendo el número óptimo 5 directores. Dalton (1998) no encuentra evidencia significativa entre el tamaño del directorio y el valor de empresas.

A pesar de la escasa evidencia empírica en Chile, en lo que respecta al efecto del tamaño de directorio en el valor de la empresa, nuestra última hipótesis de investigación es:

H6: "Se espera una relación positiva entre el tamaño del directorio y el valor de empresa".

\section{MUESTRA, VARIABLES Y METODOLOGÍA}

\subsection{Muestra}

La muestra consiste en un conjunto de empresas no financieras, cotizadas en la Bolsa de Comercio de Santiago, durante el periodo comprendido 2006 - 2013. La información se obtuvo de la base de datos Economatica, que permitió obtener los estados financieros anuales de las empresas.

También se incorporó en la base de datos, información sobre la participación de las AFP en el directorio de las empresas. Esta información, pública, se encuentra disponible en la web de la Superintendencia de Pensiones (SP)7. Por último para extraer la información respecto al directorio y grupos económicos de las empresas se obtuvo la información en la Superintendencia de Valores y Seguros (SVS) y en las respectivas memorias anuales.

Como resultado de la recolección de información se ha conformado un panel no balanceado, compuesto por 789 observaciones, correspondiente a 85 empresas chilenas, sociedades anónimas abiertas no financieras.

A objeto de mejorar la calidad de la información se eliminaron aquellas empresas que no presentan información sobre Q Tobin durante 3 periodos seguidos.

\subsection{Variables}

Se ha considerado como variable dependiente la Q Tobin (Morck 1988, McConnell y Servaes 1990, Bebchuk y Cohen 2009), como proxy del desempeño de la empresa. Esta variable Q Tobin compara el valor de mercado de la empresa con el costo de reposición de sus activos (Tobin, 1969).

Brealey y Myers (1993) mencionan diferencias importantes en el ratio Q. En el numerador incluye todos los títulos de deuda y acciones de la empresa, no sólo las ordinarias. El denominador incluye todos los activos, activos que no están valorados al costo de adquisición, como en la contabilidad de la empresa, sino a lo que costaría reponerlos.

Con lo señalado anteriormente, en este trabajo hemos calculado la Q Financiera (1), como Proxy de la Q Tobin, siguiendo Chung y Pruitt (1994):

$$
\text { QTobin }(1)=\frac{\text { Valor_Libro_Deuda }+ \text { Valor_Mercado_Patrimonio }}{\text { Valor_Libro_Activos_Totales }}
$$

Esta aproximación de la Q Tobin, es comparada con la Q Tobin de Linderberger y Ross (1981), concluyendo que la medida de Chung y Pruitt (1994) explica por lo menos un 96.6\% de la Q Tobin.

Como variables explicativas hemos considerado la estructura de propiedad. PROP1, PROP3 y PROP5 las que consideran el porcentaje de propiedad del principal accionista, de los tres primeros accionistas y de los 5 primeros accionistas, respectivamente (Espinosa 2009, Margaritis y Psillaki 2010).

Como hemos visto, el problema de agencia se presenta de distinta manera para los diferentes niveles de concentración de propiedad. Con la finalidad de medir el efecto de la pertenencia de una empresa en algún conglomerado, hemos definido una variable dicotómica: grupos económicos (GE) (Raineri y Valenzuela 2001, Silva et al 2006), que toma valor 1 si la empresa pertenece a algún grupo económico, y 0 en caso contrario.

Por su parte, para analizar el efecto de los inversionistas institucionales, en particular fondos de pensiones, sobre el valor de la empresa hemos definido la variable AFP como el porcentaje de propiedad en manos de la AFP. Por otro lado, DAFP será una variable dicotómica que revela el derecho de la AFP a elegir director. 
Para medir el efecto del directorio sobre el valor de la empresa hemos definido, INDEP como la proporción de directores independientes (Lefort y Urzua, 2008) y tamaño del directorio QDIR, como el número total de los directores en una empresa.

\section{Variables de control}

Por último, se ha incluido algunas variables de control, cuya incorporación se fundamenta en el efecto que genera la deuda en el valor de la empresa.

Para la estructura de capital, hemos incorporado LEV como el cociente entre el valor libro de la deuda y el patrimonio. Dlp, como proxy del endeudamiento de largo plazo, medido a través del cociente de los pasivos no corrientes y el valor total de la deuda.

Para incluir el efecto del tamaño de la empresa se incorporó TAM, se ha calculado como el logaritmo natural del total activos. Para medir el efecto de la rentabilidad de la empresa incluimos la rentabilidad operacional (ROA) como el cociente entre la utilidad operacional y total activos. Finalmente como variable proxy de tangibilidad de los activos, calculamos TANG como la relación entre propiedad, planta y equipo y el total activos.

La tabla 3 explica con mayor detalle estas variables.

\section{Tabla 3: Descripción de variables}

\begin{tabular}{l|l|l}
\hline \multicolumn{1}{|c|}{ Abreviación } & \multicolumn{1}{c}{ Variable } & \multicolumn{1}{c}{ Descripción } \\
\hline Variable dependiente & $\begin{array}{l}\text { Valor libro de la deuda más valor mercado del patrimonio sobre activos } \\
\text { totales }\end{array}$ \\
\hline QTOBIN $\quad$ Creación de valor &
\end{tabular}

\section{Variables Independientes}

Estructura de Propiedad

\begin{tabular}{l|l|l|}
\hline PROP1 & Concentración & Concentración de la propiedad en manos del principal accionista \\
\hline PROP3 & Concentración & Concentración de la propiedad en manos de los 3 principales accionistas \\
\hline PROP5 & Concentración & Concentración de la propiedad en manos de los 5 principales accionistas \\
\hline GE & Grupos Económicos & $\begin{array}{l}\text { Variable dicotómica; } 1 \text { empresa pertenece a un grupo económico y 0 en } \\
\text { caso contrario }\end{array}$ \\
\hline DAFP & Inversionista Institucional & Variable dicotómica; 1 si elige director y 0 en caso contrario \\
\hline AFP & Inversionista Institucional & Propiedad en manos de las AFPs \\
\hline Directorio & Directores independientes & Proporción de directores independientes \\
\hline INDEP & Tamaño del directorio & Número total de directores \\
\hline QDIR & Retorno sobre los activos & Utilidad operacional sobre valor libro de activos \\
\hline Variables de control & Tamaño de la empresa & Logaritmo natural de los activos totales \\
\hline ROA & Tangibilidad de los & Propiedad, planta y equipo sobre activos totales \\
\hline TAM & activos & Valor libro de la deuda sobre patrimonio \\
\hline TANG & Endeudamiento & Valor deuda largo plazo sobre total activos totales. \\
\hline LEV & Deuda Largo plazo & \\
\hline Dlp & &
\end{tabular}

\subsection{Metodología}

Este estudio analiza las variables que inciden en el valor de las empresas no financieras chilenas, donde QTobin es la variable a explicar y como variables explicativas tenemos una serie de proxys referidas a la estructura de propiedad (PROP1, PROP3, PROP5, GE, DAF, AFP) y del Directorio (QDIR, INDEP). Hemos definido, además, una serie de variables de control (ROA, TAM, TANG, LEV, Dlp). Lo anterior, se refunde en la ecuación (1):

$$
\begin{gathered}
\text { QTobin }_{i t}=\alpha_{i t}+\beta_{1} \operatorname{Prop}_{1-3-5 i t}+\beta_{2} \text { DummGE }_{i t}+\beta_{3} \text { DummDAFP }_{i t}+\beta_{4} A F P_{i t}+\beta_{5} I_{N D E P_{i t}}+\beta_{6} \text { QDIR }_{i t}+ \\
\beta_{7} \text { ROA }_{i t}+\beta_{8} \text { TAM }_{i t}+\beta_{9} T A N G_{i t}+\beta_{10} \operatorname{Lev}_{i t}+\beta_{11} \operatorname{Dlp}_{i t}+\varepsilon_{i t}(1)
\end{gathered}
$$

Con $\mathrm{i}=1, \ldots . ., \mathrm{N}$ y $\mathrm{t}=1, \ldots \ldots ., \mathrm{T}$ 
Donde i se refiere a la empresa, $t$ a la dimensión temporal y $\boldsymbol{\varepsilon}_{i t}$ corresponde al error estocástico.

La ecuación (1) se empleó utilizando la metodología datos de panel, con el propósito de capturar la mayor información posible en cuanto al comportamiento de las variables. Los datos de panel poseen un mayor contenido informativo, mayor variabilidad, más grados de libertad y mayor eficiencia (Baltagi, 1995).

A partir del modelo general, se estimaron modelos con datos de panel trabajando con efectos fijos y efectos aleatorios. Para resolver respecto de lo apropiado de uno u otro modelo se empleó el test de Hausman. Los resultados indican la existencia de efectos fijos, por lo que se utilizó aquel método.

\section{RESULTADOS}

\subsection{Análisis Descriptivo}

La tabla 4 muestra la estadística descriptiva para las variables empleadas en este estudio.

Observamos que la Q Tobin es de 1,51 veces, superior al 1,1 veces reportado por Lefort y Urzua (2008), al 0,97 veces reportado por Espinosa (2009), al 1,38 veces reportado por Andrés et al (2004) y 1,01 veces reportado por Silva et al (2006).

Se observa que la concentración de la propiedad cuando está en manos de un sólo accionista es de un 44,9\%, resultado similar para estudios previos (De Andrés et al 2004; Espinosa y Maquieira 2010; Espinosa 2009; Espinosa y Maquieira 2010; Lefort y Walker 2000).

Cuando la concentración de la propiedad está en manos de los tres principales accionistas, ésta aumenta a un 65.1\% igualmente similar a investigaciones previas para el caso chileno (Espinosa y Maquieira 2010; Espinosa y Maquieira 2010; Espinosa 2009; Lefort y Walker 2000).

Respecto a la concentración de la propiedad cuando está en manos de los cinco principales accionistas, esta aumenta a un 72.9\%.

En el caso de los inversionistas institucionales, en particular las administradoras de fondos de pensiones (AFP), estas poseen una participación de la propiedad en las empresas chilenas de un 2.51\% y en un $49.42 \%$ de los casos las AFP tienen derecho a elegir director.

Por otra parte, podemos observar que un 66.7\% de las empresas chilenas pertenecen a algún grupo económico (Silva et al 2006; Lefort y Urzua 2008).

La razón deuda a patrimonio (LEV) de las empresas de la muestra es de 1.203 veces, inferior al 1.6 veces reportado por Lefort y Urzua (2008) y superior al 0.57 veces al reportado por Silva et al (2006). Podemos apreciar que las empresas están endeudas en mayor proporción al corto plazo, pues la razón deuda largo plazo (Dlp) es de un 23.26\%. En el caso de la rentabilidad de los activos, podemos apreciar un ROA de un 7.42\%, superior al 4\% reportado por Silva et al (2006).

La tabla 5 muestra la matriz de correlación entre las variables de estudio. La Q Tobin presenta una relación negativa entre PROP1 (-0.0435) y significativa con PROP3 (-0.1412) y PROP5 (-0.1856), concordante con lo reportado con Espinosa y Maquieira (2010). Asimismo se constata una fuerte correlación positiva y significativa con el ROA (0.2482), tamaño del directorio QDIR (0.0908) y la tangibilidad de los activos (0.2044). Respecto al endeudamiento (LEV) se correlaciona negativamente (-0.1302).

En cuanto al tamaño (TAM) se observa una fuerte, negativa y significativa correlación con el desempeño de la empresa (-0.2619), coherente con lo reportado por Espinosa (2009). Además el tamaño de la firma, está correlacionado positiva y significativamente (0.3591) con la pertenencia a algún grupo económico (GE) y la rentabilidad operacional ROA (0.1252). Por lo que respecta la estructura capital encontramos evidencia significativa y positiva con el leverage (0.2221) y el financiamiento a través de la deuda de largo plazo (0.3191).

En lo que respecta a las AFP, y como era de esperar, existe una correlación positiva entre la propiedad en manos de los fondos de pensiones (AFP) y el porcentaje de directores independientes INDEP (0.2624). También es positivo el tamaño de la empresa TAM (0.3068). Ambos valores son estadísticamente significativos.

\section{Tabla 4: Estadísticas Descriptivas}

La tabla 4 muestra el número de observaciones, media, desviación estándar, valor mínimo y máximo para cada variable de la muestra. Las variables son las siguientes: Variable dependiente Q Tobin, es medida como valor libro de la deuda más valor mercado del patrimonio sobre activos totales. Variables independiente; la concentración de la propiedad de los accionistas mayoritarios es medido a través de PROP1 concentración en manos del principal accionista, PROP3 concentración en manos de los 3 principales accionistas y finalmente PROP5 concentración en manos de los 5 principales accionistas. Si la empresa pertenece algún grupo económico es medido a través de una variable dicotómica GE. Respecto a los inversionistas institucionales, Fondos de Pensiones, es medido a través del porcentaje en mano de las AFP a través de la variable AFP y por ultimo si la AFP elige un director en la empresa es medido como una variable dicotómica DAFP. Para medir el efecto del directorio en el desempeño de la empresa hemos estimado el tamaño del directorio QDIR medido a través del número total de 
directores y medimos la relación de directores independientes sobre el total de directores a través de INDEP. Como variables de control hemos definido, ROA como la utilidad operacional sobre valor libro de activos, el tamaño de la empresa es medido como el valor libro del total activos en millones de pesos TAM, para la tangibilidad de los activos hemos aproximado la variable TANG como la relación entre propiedad, planta y equipo sobre el total de activos. Además hemos incluido medidas acerca estructura de capital, el efecto leverage es medido como el valor libro de la deuda sobre patrimonio LEV y por último el financiamiento de los activos con deuda de largo plazo es medido por Dlp.

Tabla 4

\begin{tabular}{|c|c|c|c|c|c|}
\hline Variable & Obs. & Media & Desv. Tip. & Min. & Max. \\
\hline Q TOBIN & 789 & 1.5104 & 1.2353 & 0.1277 & 11.4378 \\
\hline PROP1 & 721 & 0.4492 & 0.2224 & 0.0555 & 0.9964 \\
\hline PROP3 & 721 & 0.6516 & 0.1985 & 0.1141 & 1.0000 \\
\hline PROP5 & 721 & 0.7293 & 0.1758 & 0.1307 & 1.0000 \\
\hline GE & 774 & 0.6679 & 0.4712 & 0.0000 & 1.0000 \\
\hline DAFP & 518 & 0.4942 & 0.5004 & 0.0000 & 1.0000 \\
\hline AFP & 789 & 0.0251 & 0.0413 & 0.0000 & 0.2092 \\
\hline INDEP & 738 & 0.4062 & 0.3251 & 0.0000 & 1.0000 \\
\hline QDIR & 1266 & 4.6011 & 0.0552 & 0.0000 & 15.000 \\
\hline ROA & 789 & 0.0742 & 0.1124 & -0.5163 & 1.1927 \\
\hline TAM & 1271 & 672,467 & 49,033 & 7.0000 & $15,177,664$ \\
\hline TANG & 789 & 1.7944 & 32.4996 & 0.0000 & 912.96 \\
\hline LEV & 789 & 1.2031 & 1.4996 & -13.8809 & 13.5395 \\
\hline Dlp & 789 & 0.2326 & 0.1416 & 0.0000 & 0.9326 \\
\hline
\end{tabular}

La tabla 5 presenta los valores correspondientes a la correlación entre las variables y bajo éstas se encuentra su significancia estadística.

Tabla 5: Matriz de Correlación

\begin{tabular}{|c|c|c|c|c|c|c|c|c|c|c|c|c|c|c|}
\hline & Q TOBIN & PROP1 & PROP3 & PROP5 & GE & DAFP & AFP & LEV & Dlp & INDEP & QDIR & $\mathrm{ROA}$ & TAM & TANG \\
\hline Q TOBIN & 1.0000 & & & & & & & & & & & & & \\
\hline \multirow{2}{*}{ PROP1 } & -0.0435 & 1.0000 & & & & & & & & & & & & \\
\hline & 0.2345 & & & & & & & & & & & & & \\
\hline \multirow{2}{*}{ PROP3 } & -0.1412 & 0.8649 & 1.0000 & & & & & & & & & & & \\
\hline & 0.0001 & 0.0000 & & & & & & & & & & & & \\
\hline \multirow{2}{*}{ PROP5 } & -0.1856 & 0.7903 & 0.9742 & 1.0000 & & & & & & & & & & \\
\hline & 0.0000 & 0.0000 & 0.0000 & & & & & & & & & & & \\
\hline \multirow{2}{*}{ GE } & -0.1279 & 0.2048 & 0.1202 & 0.0787 & 1.0000 & & & & & & & & & \\
\hline & 0.0004 & 0.0000 & 0.0014 & 0.0363 & & & & & & & & & & \\
\hline \multirow{2}{*}{ DAFP } & 0.0613 & -0.0054 & 0.0126 & 0.0086 & -0.0148 & 1.0000 & & & & & & & & \\
\hline & 0.1633 & 0.9056 & 0.7833 & 0.8505 & 0.7374 & & & & & & & & & \\
\hline \multirow{2}{*}{ AFP } & -0.0491 & -0.0186 & -0.1161 & -0.1673 & 0.2127 & 0.0281 & 1.00000 & & & & & & & \\
\hline & 0.1683 & 0.6173 & 0.0018 & 0.0000 & 0.0000 & 0.5231 & & & & & & & & \\
\hline \multirow{2}{*}{ LEV } & -0.1302 & -0.1182 & -0.0888 & -0.0821 & .0 .0243 & 0.0438 & -0.0704 & 1.0000 & & & & & & \\
\hline & 0.0002 & 0.0015 & 0.0170 & 0.0276 & 0.4999 & 0.3202 & 0.0480 & & & & & & & \\
\hline \multirow{2}{*}{ Dlp } & -0.0810 & -0.1039 & -0.0630 & -0.0539 & 0.0491 & 0.0383 & 0.0786 & 0.1949 & 1.0000 & & & & & \\
\hline & 0.0229 & 0.0052 & 0.0910 & 0.1481 & 0.1726 & 0.3844 & 0.0272 & 0.0000 & & & & & & \\
\hline \multirow{2}{*}{ INDEP } & -0.0071 & -0.3482 & -0.4101 & -0.4036 & 0.1228 & -0.0279 & 0.2624 & -0.0308 & 0.1033 & 1.0000 & & & & \\
\hline & 0.8478 & 0.0000 & 0.0000 & 0.0000 & 0.0008 & 0.5340 & 0.0000 & 0.4027 & 0.0050 & & & & & \\
\hline \multirow{2}{*}{ QDIR } & 0.0908 & 0.0778 & 0.1203 & 0.1263 & -0.0519 & 0.1029 & -0.0724 & 0.0123 & -0.0277 & -0.2634 & 1.0000 & & & \\
\hline & 0.0136 & 0.0431 & 0.0017 & 0.0010 & 0.1595 & 0.0216 & 0.0493 & 0.7378 & 0.4522 & 0.0000 & & & & \\
\hline
\end{tabular}




\begin{tabular}{|c|c|c|c|c|c|c|c|c|c|c|c|c|c|c|}
\hline & Q TOBIN & PROP1 & PROP3 & PROP5 & GE & DAFP & AFP & LEV & Dlp & INDEP & QDIR & $\mathrm{ROA}$ & TAM & TANG \\
\hline \multirow{2}{*}{$\mathrm{ROA}$} & 0.2482 & 0.2621 & 0.2302 & 0.2106 & 0.0923 & -0.0122 & 0.0122 & -0.0651 & -0.1025 & -0.1412 & 0.0207 & 1.0000 & & \\
\hline & 0.0000 & 0.0000 & 0.0000 & 0.0000 & 0.0102 & 0.7826 & 0.7323 & 0.0674 & 0.0040 & 0.0001 & 0.5738 & & & \\
\hline \multirow{2}{*}{ TAI } & -0.2619 & 0.0075 & -0.0344 & -0.0767 & 0.3591 & 0.0507 & 0.3068 & 0.2221 & 0.3191 & 0.2168 & -0.1270 & 0.1252 & 1.0000 & \\
\hline & 0.0000 & 0.8405 & 0.3563 & 0.0394 & 0.0000 & 0.2492 & 0.0000 & 0.0000 & 0.0000 & 0.0000 & 0.0005 & 0.0004 & & \\
\hline \multirow{2}{*}{ TANG } & 0.2044 & -0.0618 & -0.0997 & -0.1263 & -0.0487 & -0.0327 & -0.0176 & -0.0305 & -0.0521 & -0.0471 & 0.0598 & -0.036 & -0.1326 & 1.0000 \\
\hline & 0.0000 & 0.0972 & 0.0074 & 0.0007 & 0.1762 & 0.4573 & 0.6212 & 0.3918 & 0.1437 & 0.2009 & 0.1047 & 0.3093 & 0.0002 & \\
\hline
\end{tabular}

\subsection{Análisis explicativo}

En la tabla 6 se muestran los resultados para los distintos modelos propuestos. Puede observase, en lo que respecta a la estructura de propiedad, que en los modelos 1, 2 y 3 las variables de estructura de propiedad (PROP1, PROP3 Y PROP 5) tienen una relación negativa con el valor de la empresa. Para el caso de PROP3 y PROP5 muestran una relación negativa y estadísticamente significativa, cuando la propiedad se concentra en manos de los tres principales accionistas y en manos de los cinco principales accionistas. Este resultado, es coherente con la argumentación del efecto atrincheramiento, donde los accionistas mayoritarios expropian riquezas de los accionistas minoritarios generando un impacto negativo en el valor de la empresa. Estos resultados proporcionan evidencia que apoyan la hipótesis 1, la cual indica que una alta concentración de propiedad tiene una influencia negativa sobre el valor de la empresa. (Espinosa 2009, Lefort y Walker 2005, Espinosa y Maquieria 2010b, Silva et al 2006).

En relación a los conglomerados en Chile, podemos observar que para los modelos 1, 2 y 3, la variable grupos económicos (GE) posee una relación positiva y significativa con el valor de la empresa. En este sentido, los grupos económicos siguen cumpliendo un rol importante en la economía chilena, pues la mayoría de las empresas de la muestra pertenecen a algún grupo económico y esto genera un aporte en el valor de la empresa, ya sea por la transferencia de recursos así como conocimientos, todo para maximizar el valor del holding. Estos resultados proporcionan evidencia que apoyan la hipótesis 2 (Claessens et al, 1999).

Ahora bien, en lo que respecta al directorio, sólo podemos validar la hipótesis seis. En los modelos 2 y 3 el tamaño del directorio es positivo y estadísticamente significativo con relación al valor de la empresa (Lei y Song 2013, Lefort y Urzua 2008). En este sentido la alta concentración de propiedad y las estructuras piramidales de control corporativo, generan que la mayoría de los directores sean elegidos por los controladores, es por ello que el mercado valora el mayor tamaño en un directorio, pues existe una mayor supervisión y control sobre la alta administración, de no ser así existe un riesgo de generar una dinámica de directorio similar a la de un consejo asesor del accionista controlador (Lefort, 2008).

Respecto a las hipótesis 3, 4 y 5, los resultados arrojaron que no existe evidencia significativa para validar la relación entre las variables explicativas DAFP, AFP e INDEP con la dependiente $Q$ Tobin.

Respecto a las variables de control, la rentabilidad operacional presenta una relación positiva y significativa para los modelos 1 y 2. Como era de esperar, cuando las empresas poseen una mayor rentabilidad, éstas poseen un mayor valor.

Respecto al endeudamiento de largo plazo, encontramos una relación negativa y significativa en el modelo 1. Por ello podemos deducir que las empresas que tengan un mayor financiamiento en el largo plazo presenten un menor valor, debido al mayor costo financiero de la deuda.

Finalmente hemos encontrado una relación negativa y significativa en los tres modelos para la variable tamaño (TAM), también reportado por Espinosa (2009), Espinosa y Maquieira (2010), Espinosa y Maquieira (2005) y Silva et al (2006), aunque en contraste con la relación positiva obtenida por De Andrés et al (2004). Las empresas de mayor tamaño presentan un menor valor, ya que poseen menores oportunidades de crecimiento pues han alcanzado un estado madurez.

La tabla 6 proporciona el resultado para las regresiones de las muestras donde la variable dependiente es Q Tobin. Los modelos se estiman por medio de datos de panel mediante efectos fijos. La significancia estadística de la prueba Chi es significativa al 1\%. El Modelo 1 no contiene las variables PROP3, PROP5, AFP y LEV. El Modelo 2 no contiene las variables PROP1 y PROP5, AFP y Dlp. Por último el Modelo 3 no contiene las variables PROP1, PROP3, DAFP y Dlp. La significancia estadística se registra al lado de cada valor donde, ${ }^{* * *}$ inferior al 1\%, ${ }^{* *}$ inferior al $5 \%$ y *inferior al $10 \%$. 


\section{Tabla 6: Determinantes de la Creación de Valor}

\begin{tabular}{|c|c|c|c|}
\hline Dependiente & $\begin{array}{l}\text { Q Tobin } \\
\text { Modelo1 } \\
\text { Coeficiente } \\
\text { Desv. Tip }\end{array}$ & $\begin{array}{c}\text { Q Tobin } \\
\text { Modelo2 } \\
\text { Coeficiente } \\
\text { Desv. Tip }\end{array}$ & $\begin{array}{c}\text { Q Tobin } \\
\text { Modelo } 3 \\
\text { Coeficiente } \\
\text { Desv. Tip }\end{array}$ \\
\hline \multirow{2}{*}{ Intercepto } & $13.2341^{* * *}$ & $16.4164^{* * *}$ & $17.5116^{* * *}$ \\
\hline & 1.6505 & 1.8705 & 1.5871 \\
\hline \multirow{2}{*}{ PROP1 } & -0.2518 & & \\
\hline & 0.3862 & & \\
\hline \multirow{2}{*}{ PROP3 } & & $-0.7488^{*}$ & \\
\hline & & 0.4263 & \\
\hline \multirow{2}{*}{ PROP5 } & & & $-1.2573^{*}$ \\
\hline & & & 0.4612 \\
\hline \multirow{2}{*}{ GE } & $0.3193^{* *}$ & $0.3136^{*}$ & $0.3635^{* *}$ \\
\hline & 0.1568 & 0.1662 & 0.1507 \\
\hline \multirow{2}{*}{ DAFP } & 0.0052 & 0.0212 & \\
\hline & 0.0527 & 0.0559 & \\
\hline \multirow{2}{*}{ AFP } & & & -0.3258 \\
\hline & & & 1.2304 \\
\hline \multirow{2}{*}{ INDEP } & -0.0976 & -0.1033 & 0.1350 \\
\hline & 0.1476 & 0.1607 & 0.1520 \\
\hline \multirow{2}{*}{ QDIR } & 0.0986 & $0.1890^{*}$ & $0.1840^{* *}$ \\
\hline & 0.0964 & 0.1010 & 0.0899 \\
\hline \multirow{2}{*}{$\mathrm{ROA}$} & $0.8699^{* *}$ & $0.8520^{*}$ & 0.4654 \\
\hline & 0.4137 & 0.4389 & 0.3524 \\
\hline \multirow{2}{*}{ TAM } & $-0.5603^{* * *}$ & $-0.7460^{* * *}$ & $-0.8334^{* * *}$ \\
\hline & 0.0801 & 0.0901 & 0.0774 \\
\hline \multirow{2}{*}{ TANG } & 0.0018 & -0.0072 & -0.0011 \\
\hline & 0.0234 & 0.0249 & 0.0007 \\
\hline \multirow{2}{*}{ LEV } & & 0.0204 & 0.0163 \\
\hline & & 0.0298 & 0.0242 \\
\hline \multirow{2}{*}{ Dlp } & $-3.0990^{* * *}$ & & \\
\hline & 0.4423 & & \\
\hline $\mathrm{R} 2$ & 0.2884 & 0.2003 & 0.1965 \\
\hline Obs & 463 & 463 & 675 \\
\hline Prob F (statistic) & 0.0000 & 0.0000 & 0.0000 \\
\hline
\end{tabular}

\section{CONCLUSIONES}

Este trabajo investiga la relación existente entre el valor de empresa y características internas de gobierno corporativo, por medio de un panel no balanceado de 85 empresas chilenas no financieras, cotizadas en la Bolsa de Comercio de Santiago.

Por medio de la presente se pudo constatar la alta concentración de la propiedad de las empresas chilenas. De acuerdo a los antecedentes disponibles, se puede establecer que cuando la propiedad está en manos del principal accionista, este concentra más del 45\% de ella, cifra que aumenta al 72\% cuando sus dueños son los cinco principales accionistas.

Esta alta concentración de la propiedad, acentúa el conflicto agencia entre accionistas mayoritarios y minoritarios, lo que afecta negativamente el desempeño de las compañías. Los resultados parecen indicar que a mayor concentración de la propiedad, disminuye el valor de las empresas.

Como se mencionó, la escasa protección legal a los accionistas genera imperfecciones en el mercado chileno y motiva a la concentración de la propiedad. En países como Estados Unidos e Inglaterra existe una alta protección legal a los inversionistas minoritarios, lo que genera mayor profundidad y eficiencia en el mercado de capitales, no así en los mercados Latinoamericanos, que aún se encuentran en etapas tempranas del desarrollo de sus mercados de capitales. 
Por tanto, la concentración de propiedad y el bajo resguardo legal genera expropiación de riquezas mediante tunneling ${ }^{8}$, desde los accionistas mayoritarios a los minoritarios. Nuestros resultados, por tanto, permiten establecer que una alta concentración de la propiedad tiene una influencia negativa en el valor de la empresa.

Ahora bien, la alta concentración de la propiedad y pertenencia a grupos económicos (más de un 66\% de la muestra) es una realidad para las empresas del mercado chileno. A modo de ejemplo se citan los siguientes: grupo Angelini, grupo Matte, grupo Solari y grupo Luksic, todos vinculados a familias de estos apellidos. Los resultados de nuestra investigación nos permiten validar nuestra segunda hipótesis y establecer que la pertenencia de la empresa en un grupo económico tiene una influencia positiva en el valor de la empresa. Las empresas al interior del conglomerado pueden transferir recursos y habilidades, generando sinergias tanto operacionales como financieras, todo lo cual beneficia al grupo empresarial.

Hay que destacar que en Chile, los principales inversionistas minoritarios son las AFP. De ahí la importancia de un adecuado marco regulatorio e institucional, que garantice la protección de los fondos de todos los afiliados. Sin embargo, no hemos encontrado evidencia empírica que nos haga validar las hipótesis relacionadas con las AFP. Sin embargo, respecto al directorio hemos encontrado evidencia empírica que nos permite establecer que existe una relación positiva entre tamaño del directorio y el valor de empresa.

Resumidamente, las empresas con mejor desempeño en Chile pertenecen a algún grupo económico, con un número elevado de directores y con una menor concentración de propiedad.

Hemos de distinguir, además, como futura línea investigación la incorporación de otras variables relativas al directorio de las empresas, por ejemplo, la existencia de directores profesionales, así como la compensación al directorio. La evidencia en Chile respecto de este tema es escasa, por lo que su análisis reviste un tremendo valor. Así mismo, la incorporación de información de otras economías de la región, aporte una mayor riqueza al análisis, puesto que a nivel Latinoamericano existe poca evidencia aún.

La solución a la problemática sobre gobiernos corporativos parece estar enfocada en un monitoreo constante y eficaz por parte del directorio de las empresas, mayor transparencia en la información y un mejor sistema legal, con normas y leyes que brinden la protección necesaria a todo tipo de inversionistas.

a. Autor de correspondencia

1. Casos de empresas multinacionales con problemas en sus gobiernos corporativos.

2. Compañía estadounidense creada en 1850 de servicios globales financieros, que a partir de la crisis subprime quiebra y se declara en bancarrota el año 2008. La investigación determinó que existieron malas prácticas de sus directores ya que redujeron su apalancamiento de manera fraudulenta.

3. Costos de supervisión, costos de formalización y pérdida residual. Jensen y Meckling (1976).

4. Doing Business 2011

5. http://www.economiaynegocios.cl/noticias/noticias.asp?id=100017

6. Informe Valor y Rentabilidad de los Fondos de Pensiones, Mayo 2014. SAFP.

7. Informe “Participación de las AFP y la AFC en juntas y asambleas” años 2006 - 2013.

8. Cuando hay accionistas controladores, el riesgo, llamado "tunneling" (túnel) en la literatura internacional, se refiere a que los controladores buscan su propio beneficio a expensas de los otros accionistas. 


\section{REFERENCIAS BIBLIOGRÁFICAS}

Aparicio, C. (2006). La relación entre el valor de la empresa y su estructura accionarial en España. Comisión Nacional del Mercado de Valores (18)

Asociación de AFP. (Mayo de 2008). AFP defienden los Intereseses de los Accionsitas Minoritarios y de los Afiliados . Serie de Estudios(66). Chile.

Banco Mundial (2011). Doing Business 2011: Creando oportunidades para los emprendedores. Recuperado a partir de: http:// espanol.doingbusiness.org//media/WBG/DoingBusiness/Documents/Annual-Reports/Overview/DB11-Overview-Spanish.pdf

Baltagi, B. (1995). Econometric Analysis of Panel Data. New York, NY: John Wiley and Sons.

Bebchuk, L., Cohen, A., \& Ferrell, A. (2009). What matters in corporate governance? Review of Financial Studies, 22(2), $783-827$.

Berle, A., \& Means, G. (1932). The modern corporation and private property. New York, NY: McMillan.

Boubaker, S. (2007). Ownership-control discrepancy and firm value: Evidence from France. Multinational Finance Journal, 11, $211-252$.

Brealy, R., \& Myers. S. (1993). Fundamentos de Financiación Empresarial (4ta ed.). Madrid: McGraw Hill Interamericana.

Casassus, J., Ferrando, J., \& Huidobro, R. (2010). Gobiernos Corporativos. La Clase Ejecutiva. Santiago, Chile: Universidad Católica y El Mercurio.

Castro, M. S., Brown, C. J., \& Báez-Díaz, A. (2009). Prácticas de gobierno corporativo en América Latina. Academia-Revista Latinoamericana de Administración, 43, 26-40.

Claessens, S., Djankov, S., \& Klapper, L. (2000). The role and functioning of business groups in east Asia and Chile. Revista Abante, 3(1), 97-107.

Chong, A., \& López de Silanes, F. (2007, marzo). Corporate governance in Latin America. (IDB Working Paper No 494). Recuperado a partir de: https://papers.ssrn.com/sol3/papers.cfm?abstract_id=1820067

Chung, K. H., \& Pruitt, S. W. (1994). A simple approximation of Tobin's q. Financial Management, 23(3), 70-74.

Dalton, D. R., Daily, C. M., Ellstrand, A. E., \& Johnson, J. L. (1998). Meta-analytic reviews of board composition, leadership structure, and financial performance. Strategic Management Journal, 19, 269-290.

De Andrés, P., San Martín, P., \& Saona, P. (2004). Decisiones financieras en la empresa chilena: una mirada a través de las oportunidades de crecimiento. Revista Abante, 7(1), 3-34

Dechow, P., Sloan, G., \& Sweeney, A. (1996). Causes and consequences of earnings manipulation: An analysis of firms subject to enforcement actions by the SEC. Contemporary Accounting Research, 13, 1-36.

Demsetz, H., \& Lehn, K. (1985). The structure of corporate ownership: Causes and consequences. The Journal of Political Economy, 93(6), 1155-1177.

Espinosa, C. (2009). Estructura de propiedad y desempeño de la firma: evidencia para Chile. Academia Revista Latinoamericana de Administración, 43, 1-19.

Espinosa, C., \& Maquieira, C. (2010, octubre). Diversificación y desempeño en Sud América: evidencia para Chile (Documento de Trabajo 10). Recuperado a partir de la web de la Facultad de Economía y Empresa de la Universidad Diego Portales: http:// economiayempresa.udp.cl/documentos-de-trabajo/

Espinosa, C. \& Maquieira, C. (2010b). Desempeño y concentración de la propiedad en empresas chilenas emisoras de American Depositary Receipts (ADR). Cuadernos de Administración, 23(40), 95-116

Fama, E., \& Jensen, M. (1983). Separation of ownership and control. The Journal of Law \& Economics, 26(2), 301-325.

Fuerst, O., \& Kang, S. H. (2004). Corporate governance, expected operating performance, and pricing. Corporate Ownership and Control, 1(2), 13-30

Hart, O. (1983). The market mechanism as an incentive scheme. The Bell Journal of Economics, 14(2), 366-382.

Hart, O. (1995). Corporate governance: some theory and implications. Economic Journal, 105(430), 678-689.

Hölmstrom, B. (1979). Moral hazard and observability. The Bell Journal of Economics, 10(1), 74-91.

Jensen, M. (1986). Agency cost of free cash flow, corporate finance, and takeovers. American Economic Review, 76(2), 323-329.

Jensen, M., \& Meckling, W. (1976). Theory of the firm: managerial behavior, agency costs, and ownership structure. Journal of Financial Economics, 3(4), 305-360.

John, K., \& Senbet, L. W. (1998). Corporate governance and board effectiveness. Journal of Banking \& Finance, 22, 371-403. 
Klein, A. (2002). Audit committee, board of director characteristics, and earnings management. Journal of Accounting and Economics, 33, 375-400.

La Porta, R., Lopez-de-Silanes, F., \& Shleifer, A. (1999). Corporate ownership around the world. The Journal of Finance, $54,471-517$.

Lefort, F. (2004). Gobierno Corporativo: ¿Qué es? y ¿Cómo andamos por casa? Cuadernos de Economía, 40, $207-237$.

Lefort, F., \& González, R. (2008). Hacia un mejor gobierno corporativo en Chile. Revista Abante, 11(1), 19-45.

Lefort, F., \& Urzúa, F., (2008). Board independence, firm performance and ownership concentration: Evidence from Chile. Journal of Business Research, 61(6), 615-622.

Lefort, F. \& Walker E. (2000). The effects of economic shocks on corporate governance systems in chile. Revista Abante, 2(2), 183206.

Lefort, F., \& Walker, E. (2005, marzo). The effect of corporate governance practices on company market valuation and payout policy in Chile (IDB Working Paper No 515). Recuperado a partir de: https://papers.ssrn.com/sol3/papers.cfm?abstract_id=676710

Lefort, F., \& Walker, E. (2007). Do markets penalize agency conflicts between controlling and minority shareholders? Evidence from Chile. The Developing Economies, 45, 283-314.

Lei, A. C., \& Song, F. M. (2012). Board structure, corporate governance and firm value: evidence from Hong Kong. Applied financial economics, 22(15), 1289-1303.

Lindenberg, E. B., \& Ross, S. A. (1981). Tobin's q ratio and industrial organization. Journal of Business, 54(1), 1-32.

Margaritis, D., \& Psillaki, M. (2010). Capital structure, equity ownership and firm performance. Journal of Banking \& Finance, 34(3), 621-632.

McConnell, J., \& Servaes, H. (1990). Additional evidence on equity ownership and corporate value. Journal of Financial Economics, 27(2), 595-612.

Morck, R., Shleifer, A., \& Vishny, R. (1988). Management ownership and market valuation: an empirical analysis. Journal of Financial Economics, 20(1), 293-315.

OECD. (2004). OECD Principles of Corporate Governance. Organisation for Economic Co-operation and Development. París: OECD Publications.

Peasnell, K.V., Pope, P.F., \& Young, S. (2001). Board monitoring and earnings management: Do outside directors influence anormal accruals? Working Paper. Lancaster University.

Pedersen, T., \& Thomsen, S. (2001). The causal relationship between insider ownership, Owner identity and market valuation among the largest european companies. Copenhagen Business School(15).

Raineri, R., \& Valenzuela, P. (2001). El valor de las estructuras de propiedad en el desempeño de las empresas chilenas (1990-2000). Segundo Encuentro de Finanzas. Santiago de Chile.

Shleifer, A. \& Vishny, R. (1997). A Survey of Corporate Governance. Journal of Finance, 52(2), 737-783.

Shleifer, A. \& Vishny, R. (1986). Large shareholders and corporate control. The Journal of Political Economy, 94(3), 461-488.

Silva, F., Majluf, N., \& Paredes, R. (2006). Ownership structure and performance: empirical evidence from Chilean firms. Corporate Ownership and Control, 3, 73-181.

Tobin, J. (1969). A general equilibrium approach to monetary theory. Journal of money, credit and banking, 1(1), 15-29.

Tong, S., \& Ning, Y. (2004). Does Capital Structure Affect Institutional Investor Choices? Journal Of Investing, 13(4), 53-56.

Walker, E., \& Lefort, F. (2003). Pension Reform and Capital Markets: Are There Any (Hard) Links? Social Protection Discussion Paper Series(201)

Xie, B., W, D., \& DaDalt, P. (2003). Earnings management and corporate governance: the role of the board and the audit committee. Journal of corporate finance, 9(3), 295-316.

Yermack, D. (1996). Higher market valuation of companies with a small board of directors. Journal of Financial Economics, 40(2), 185-211. 